\title{
PERFIL EPIDEMIOLÓGICO DO CÂNCER DE MAMA E DE OVÁRIO: ANÁLISE DE DADOS PARCIAIS NUMA COORTE REGIONAL DE PACIENTES ONCOLÓGICOS
}

Epidemiological profile of breast and ovarian cancer: partial data analysis in a regional cohort of cancer patients

\footnotetext{
Emilli Cristina Dos Santos ${ }^{1}$; Juliano Sartori ${ }^{2}$; Tayná Andressa Wencelewski1 ${ }^{1}$; Nicole De Albuquerque $^{1}$; Franciele Dartora ${ }^{1}$; Gabriela Bassani Fahl ${ }^{1}$; Rafael Rossa Marsarotto ${ }^{3}$; Paola Wozniak Ceni ${ }^{1}$; Bruna Albiero Decesaro ${ }^{1}$; Milena Bitarello ${ }^{1}$; Luma Girardi Kautz ${ }^{1}$; Amanda Peracchi Schneider ${ }^{1}$
}

${ }^{1}$ Acadêmicas do Curso de Medicina na Universidade Regional Integrada do Alto Uruguai e das Missões - Câmpus de Erechim; E-mail: emilli.cris97@gmail.com

${ }^{2}$ Médico oncologista clínico, Doutor em Gerontologia Biomédica e Professor do Curso de Medicina da Universidade Regional Integrada do Alto Uruguai e das Missões - Câmpus de Erechim;

${ }^{3}$ Acadêmico do Curso de Medicina na Universidade Regional Integrada do Alto Uruguai e das Missões - Câmpus de Erechim;

Data do recebimento: 27/09/2020 - Data do aceite: 17/03/2021

RESUMO: As neoplasias mamárias e ovarianas são extremamente frequentes na população feminina. Os gênes Breast Cancer 1 (BRCA1) e Breast Cancer 2 (BRCA2) são reguladores do ciclo celular e estão envolvidos na gênese tumoral de ambas as neoplasias. O delineamento de um perfil epidemiológico de pacientes com mutação dos genes citados é importante para o aprimoramento de estratégias terapêuticas, rastreamento precoce, intervenções redutoras de risco, promoção e prevenção da saúde. O presente artigo tem por objetivo apresentar uma breve revisão bibliográfica e os resultados clínicos e epidemiológicos parciais referentes à pesquisa acadêmica sobre o tema, desenvolvida na região nordeste do estado do Rio Grande do Sul.

Palavras-chave: Gene BRCA1. Gene BRCA2. Câncer de mama e ovários. 
ABSTRACT: Breast and ovarian neoplasms are extremely common in the female population. The genera Breast Cancer 1 (BRCA1) and Breast Cancer 2 (BRCA2) are cell cycle regulators and are involved in the tumor genesis of both neoplasms. The design of an epidemiological profile of patients with aforementioned genes mutation is extremely important for improving therapeutic strategies, early screening, risk-reducing interventions, health promotion and prevention. The aim of this article is to present a brief bibliographic review and partial clinical and epidemiological results related to academic research on the topic, developed in the northeast region of Rio Grande do Sul state.

Keywords: Gene BRCA1. Gene BRCA2. Breast and ovarian cancer.

\section{Introdução}

Os cânceres de mama e ovário, atualmente são muito incidentes nas populações mundiais. O câncer de mama, por sua vez, é a neoplasia que mais acomete a população feminina e também o de maior mortalidade entre as mulheres. Por outro lado, o câncer de ovário é um pouco menos frequente que o câncer de mama e que os demais tipos de cânceres que acometem o sistema reprodutor feminino das mulheres. O que ambas neoplasias têm em comum, em alguns casos, são as mutações encontradas nos Genes Breast cancer 1 (BRCA1), encontrado no cromossomo 17 e Breast cancer 2 (BRCA2), localizado no cromossomo 13. Esses genes possuem correlação com o desenvolvimento das neoplasias citadas, principalmente quando relacionadas a heranças familiares.

O presente artigo tem por objetivo descrever o perfil epidemiológico de pacientes portadoras de câncer de mama e de ovário de uma coorte regional de pacientes oncológicos. Os resultados são dados parciais gerados a partir de uma pesquisa com pacientes participantes do projeto de pesquisa "Perfil epidemiológico e estimativa de sobrevida em pacientes portadores de câncer de mama e/ ou câncer de ovário com mutações gênicas $B R C A 1$ e/ou BRCA2 submetidos a tratamento oncológico" realizada na Universidade Regional Integrada do Alto Uruguai e das Missões - Câmpus de Erechim. Além disto, propõe-se a apresentar um breve referencial teórico abordando aspectos sobre a epidemiologia dos cânceres de mama e de ovário, sua relação com a expressão, incidência e prevalência dos genes BRCA 1 e BRCA2, critérios clínicos de prevenção e rastreamento de pacientes portadores de mutações gênicas.

\section{Referencial Teórico}

O câncer de mama representa a neoplasia mais prevalente que acomete a população feminina mundial. Segundo dados do estudo GLOBOCAN, foram estimados cerca de 2,1 milhões de novos casos desde o ano de 2018 e segue sendo a causa mais frequente de óbitos por câncer (BRAY; FERLAY; SOERJOMATAR et al., 2018). No cenário nacional, para o ano de 2020, são esperados, segundo dados epidemiológicos do Instituto Nacional do Câncer, aproximadamente, 66 mil novos casos e, aproximadamente, 16 mil óbitos (BRASIL, 2020). Esta neoplasia possui um padrão de apresentação e crescimento heterogêneos e seu desenvolvimento é influenciado 
por diversos fatores relacionados ao estilo e hábitos de vida, obesidade, história reprodutiva e uso de terapia hormonal (BRAY; FERLAY; SOERJOMATAR et al., 2018).

A neoplasia ovariana é o segundo tipo de câncer ginecológico mais recorrente em mulheres. Mundialmente, representou cerca de 260 mil novos casos em 2018 (BRAY; FERLAY; SOERJOMATAR et al., 2018). Em relação aos dados epidemiológicos nacionais, são esperados cerca de 6 mil novos casos para o triênio 2020 a 2022. De modo geral, eles podem ser originados de células epiteliais, estromais ou de linhagem germinativa, acometem em maior porcentagem mulheres nas sexta e sétima décadas de vida. Os fatores de risco são múltiplos e dentre eles destaca-se os genéticos, com significativa influência dos genes Breast cancer 1 (BRCA1) e Breast cancer 2 (BRCA2), os hábitos de vida, sedentarismo, obesidade, uso de hormônios endógenos e história reprodutiva (BRASIL, 2020).

As neoplasias mamárias são carcinomas que podem se manifestar de forma distintas quanto ao perfil biológico de agressividade, in situ, borderline ou invasivas, sendo classificadas conforme a penetração ou não na camada basal. Quando não penetram na camada basal são denominadas in situ, sendo o carcinoma ductal in situ (subdividido em comedocarcinoma e não comedocarcinoma) e o carcinoma lobular in situ, os principais tipos histológicos desse grupo. Já o grupo dos carcinomas invasivos (também denominados infiltrantes) penetram na camada basal e são representados conforme as características moleculares. Podem ser luminais, não luminais, hiperexpressores de HER-2 (Fator de crescimento epidérmico humano) ou triplo negativos. Assim, são classificados em carcinomas RE-positivo (Receptor de estrogênio) e HER2-negativo (luminal A ou Luminais B), carcinoma HER2-positivo e carcinoma RE-negativo e HER2-negativo (triplo nega- tivos). Podem ainda ser divididos, conforme as características histológicas, em: carcinoma ductal invasivo, carcinoma lobular invasivo, carcinoma medular, carcinoma mucinoso (colóide), carcinoma tubular, carcinoma secretor, carcinoma papilífero, carcinoma micropaplífero, carcinoma apócrino, carcinoma de células fusiformes, carcinoma adenoescamoso de baixo grau e carcinoma adenóide cístico. O tumor filoide e a doença de Paget são tumores com características peculiares. O tumor filoide pode se manifestar de forma benigna, borderline ou maligna, com característica de recidiva após a excisão cirúrgica. E a doença de Paget geralmente está associada ao carcinoma ductal in situ ou ao carcinoma ductal invasivo (KUMAR; ABBAS; FAUSTO, 2010).

Em relação às neoplasias ovarianas, que também podem ser classificadas conforme o comportamento biológico (em benignas, borderline e malignas) e o tecido de origem (em tumores epiteliais, tumores de células germinativas e tumores do estroma e dos cordões sexuais) encontramos diversos tipos. Os tumores epiteliais, por exemplo, são frequentemente associados ao comportamento benigno, afetando principalmente mulheres jovens. Nesse grupo encontram-se seis tipos histológicos diferentes com origem epitelial: tumores serosos, tumores mucinosos, tumores ovarianos endometrioides, carcinoma de células claras, cistadenofibroma e tumores de células transicionais (KUMAR; ABBAS; FAUSTO, 2010).

Os tumores serosos são neoplasias císticas em que a apresentação maligna é a mais comum. São subdivididos conforme o grau de atipia nuclear, sendo carcinomas de baixo grau os tumores bem diferenciados e os tumores de alto grau são as neoplasias pouco ou moderadamente diferenciados. Já os tumores mucinosos apresentam-se, frequentemente, como benignos ou borderline, sendo que quando se tornam invasivos 
são geralmente agressivos e potencialmente fatais. Em comparação aos tumores serosos e os mucinosos, os tumores endometrioides são menos comuns, sendo distinguidos em relação a presença de glândulas tubulares. Quando apresentam características histológicas benignas são denominados de adenofibromas endometrioides, e quando malignos, são chamados de carcinomas endometrioides (caracterizam-se pela bilateralidade e se estendendo além do trato genital). Os tumores de Brenner (conhecidos também como tumores de células transicionais) são em geral unilaterais e benignos, mesmo com manifestação clínica de tumores volumosos na pelve. A variação para carcinoma de células claras e cistoadenofibroma são incomuns (KUMAR; ABBAS; FAUSTO, 2010).

As neoplasias ovarianas originadas em células germinativas também são divididas em: teratomas, disgerminoma, tumores do saco vitelínico, coriocarcinoma e outros tumores de células germinativas. Quando se apresentam como teratomas, podem ser benignos (maduros), malignos (imaturos) ou monodérmicos (altamente especializados). A grande maioria são tumores benignos e bilaterais, sendo, nestas condições, denominados de cistos dermoides. Os malignos e os mesodérmicos são raros quando se manifestam apresentam padrão invasivo e são encontrados com maior expressão em adolescentes pré-púberes e mulheres jovens (KUMAR; ABBAS; FAUSTO, 2010).

Representando o grupo mais comum de tumores malignos com origem em células germinativas encontramos os tumores do saco vitelino (também conhecidos como tumores do seio endodérmico), que afetam, principalmente, crianças e mulheres jovens. Os tumores denominados de coriocarcinoma, originados nos ovários, são, geralmente, agressivos e fatais. Entre outros tumores de células germinativas encontramos: carcinoma embrionário, poliembrioma e tumor de célu- las germinativas misto (KUMAR; ABBAS; FAUSTO, 2010).

Por fim, há os tumores originados nas células do estroma e dos cordões sexuais, sendo: os tumores de células da granulosa (que são potencialmente malignos e subdivididos em adultos e juvenil), os tumores de células de sertoli-leydig (que ocorrem em todas idades, sendo unilaterais), os tumores de células do hilo, o luteoma de gravidez,o gonadoblastoma, os fibromas, os tecomas e, finalmente, os fibrotecomas (KUMAR; ABBAS; FAUSTO, 2010).

As neoplasias de mama e ovário possuem algum nível de relação com histórico familiar no surgimento e desenvolvimento de ambas. Em sua origem mutacional estão as alterações nos genes Breast cancer $1 \mathrm{e}$ Breast cancer 2. O remodelamento de ambos os genes ainda são considerados raros, entretanto quando existentes possuem alta penetrância, resultando em uma síndrome hereditária de câncer de mama e ovários em quem possui essa condição. As mutações no gene $B R C A 1$, em geral, expressam receptores de estrogênio (ER), receptor de progesterona (RP), e HER2. Já no gene $B R C A 2$, são mais similares a tumores de aparecimento esporádico e ambos são supressores tumorais e estão envolvidos no ciclo celular. Em relação à taxa de desenvolvimento de neoplasia mamária em indivíduos com mutação em BRCA1 é cerca de $50 \%$ e em $B R C A 2$ até $15 \%$ Já em relação ao desenvolvimento de tumor de ovário $B R C A 1$, representa cerca de $40 \%$ de taxa de risco e $B R C A 2$ até 20\% (SHIOVITZ; KORDE, 2015).

Os genes BRCA1 e BRCA2 possuem envolvimento com o risco de câncer de mama herdado. Possuem diversos tipos de mutações, entretanto, as envolvidas a alelos deletérios em ambos os tipos são as mais comuns e que possuem alto risco no desenvolvimento da referida neoplasia. A correlação elevada entre o grau de expressão dos fatores gené- 
ticos e a probabilidade de desenvolvimento neoplásico, se dá pela análise de histórico familiar com presença de câncer de mama em pelo menos quatro linhagens subsequentes, inclusive com a neoplasia, estando presente em homens. A triagem genética deve ser realizada e direcionada a famílias em que a história clínica demonstre para uma possível presença de mutação, a fim de orientar a prevenção do portador da neoplasia e de linhagens posteriores. Além disso, estudos genéticos apontam a proteína reguladora do ciclo celular TP53, também mutada em alguns tipos de câncer de mama, tendo por consequência um desenvolvimento mais precoce da supracitada doença (NICE, 2013).

Estima-se que cerca de $10 \%$ ou mais dos casos de câncer de mama são relacionados à herança de mutações genéticas (DOWNS; WANG, 2015). Nesse contexto, os genes supressores tumorais mais importantes associados ao câncer de mama são BRCA1 e BRCA2, os quais estão relacionados aos aspectos centrais do metabolismo celular, tais como reparo de danos ao DNA, regulação da expressão gênica e controle do ciclo celular. (DUFLOTH; CARVALHO, HEINRICH et al., 2005; COELHO; SANTOS, CAETANO et al., 2018).

As mutações no genes BRCA1 são localizadas no cromossomo 17 e posicionadas no seu braço longo, na posição 21. Já no gene BRCA2 a mutação é localizada no braço longo do cromossomo 13, especificamente na posição 12.3, especialmente quando são identificadas no éxon 11. Pacientes que apresentam alguma das mutações ou ambas, possuem o risco aumentado do desenvolvimento de neoplasias mamárias ou ovarianas (REBBECK; MITRA, WAN et al., 2015).

Os carcinomas associados ao BRCA1 são do tipo histológico ductais infiltrantes e pouco diferenciados, os quais, frequentemente, apresentam características morfológicas do carcinoma medular típico ou atípico. Os tumores associados ao BRCA2 apresentam histologia e imunofenótipo semelhantes ao câncer de mama esporádico. (HONRADO; BENÍTEZ; PALACIOS, 2006; SØNDERSTRUP; JENSEN; EJLERTSEN et al., 2019). Ademais, o câncer de mama com mutação no BRCA1, geralmente, exibe o fenótipo do câncer de mama triplo-negativo, o qual é definido por níveis indetectáveis de receptor de estrogênio, receptor de progesterona e receptor de fator de crescimento epidérmico humano 2 (HER2). Mutações no BRCA1 ocorrem em $8,5 \%$ a $28 \%$ das pacientes com câncer de mama triplo-negativo. (MORI; KUBO; VELASQUEZ et al., 2018)

As mulheres já diagnosticadas com câncer de mama correm um risco muito maior de desenvolverem um segundo câncer de mama se portadoras de uma mutação no BRCA1 ou BRCA2. As mutações no BRCA1 conferem um risco de $64 \%$ de câncer na mama contralateral aos 70 anos, ou $20 \%$ dentro de cinco anos após o diagnóstico inicial, enquanto as mutações no BRCA2 aumentam esses riscos para cerca de $50 \%$ aos 70 anos ou $12 \%$ dentro de cinco anos após o diagnóstico do primeiro câncer de mama. Os carcinomas hereditários de mama atribuíveis às mutações no BRCA1 e BRCA2 possuem as suas próprias morfologias e características imunoistoquímicas (SØRENSEN, 2019)

Os tumores ovarianos carregam em sua gênese a heterogeneidade, o que divide sua classificação nos tipos epiteliais e não epiteliais. Esses critérios levam em consideração os mecanismos envolvidos no desenvolvimento carcinogênico, tendo por base os genes mutados, principalmente $B R C A 1$ e $B R C A 2$ (LEDERMANN; RAJA; FOTOPOULOU et al., 2013).

Os cânceres ovarianos de linhagem epitelial apresentam classificação baseada em critérios histológicos, sendo considerados carcinomas serosos de alto grau e de baixo grau, de células claras, endometrioides 
e mucinosos. Em aproximadamente 15\% das pacientes há susceptibilidade herdada, relacionada, majoritariamente, à linhagem germinativa dos genes BRCA1 e BRCA2 (MOSCHETTA; GEORGE; KAYE et al., 2016). O risco cumulativo para desenvolvimento da neoplasia foi estimada até os 80 anos e estimou-se cerca de $40 \%$ de risco para presença de mutação em BRCA1 e $17 \%$ se mutação em BRCA2 (KUCHENBAECKER; HOPPER; BARNES et al., 2017).

Por estarem relacionados à regulação do ciclo celular, esses genes são considerados mediadores clinicamente relevantes da instabilidade genômica, sendo seu potencial reparador o de maior interesse na gênese do câncer de ovário. É descrito na literatura que a maior parte das mutações dessa linha germinativa está relacionada ao tipo histológico seroso de alto grau, apesar de existirem relatos em outros subtipos histológicos, como em células endometrioides claras (MOSCHETTA; GEORGE; KAYE et al., 2016).

Para o diagnóstico de cânceres hereditários de mama e ovário relacionados com os genes BRCA1 e BRCA2, o foco principal é o aconselhamento genético, para definir indivíduos e famílias de alto risco e, se for o caso, a investigação das mutações nesses genes poderá ser indicada. Esse exame para avaliação genética é considerado o padrão -ouro para o diagnóstico de pacientes com síndromes hereditárias. Para isso, segundo Lancaster (2007), são considerados pacientes com risco superior a 20-25\% de apresentar predisposição herdada para câncer de mama e de ovário para as quais se recomenda avaliação de risco genético, os seguintes achados: mulheres com antecedente pessoal de câncer de mama e de ovários; mulheres com câncer de ovário e uma familiar próxima $\left(1^{\circ}, 2^{\circ}\right.$ e $3^{\circ}$ graus) com câncer de mama surgido com idade $\leq 50$ anos ou câncer de ovário em qualquer idade; mulheres com câncer de mama em qualquer idade que tenham ancestrais Ashke- nazi; mulheres com câncer de mama em idade $>50$ anos e uma familiar próxima $\left(1^{\circ}, 2^{\circ} \mathrm{e}\right.$ $3^{\circ}$ graus) com câncer de ovário ou familiar próximo do sexo masculino com câncer de mama em qualquer idade (LANCASTER; POWELL; KAUFF et al., 2007. p. 160).

O principal objetivo do teste genético é a identificação de mulheres com mutações deletérias dos genes BRCA1 e BRCA2, para intervenção por meio de cirurgias profiláticas, com o objetivo de evitar o desenvolvimento do câncer (COMANDER; CYGLER; RYAN; 2015). A cirurgia profilática de salpingo-ooforectomia (SOOR), atualmente é recomendada para os casos de mutação nos genes BRCA. A salpingo-ooforectomia bilateral se justifica por existirem evidências de que, somente o acompanhamento de pacientes que têm a mutação nos genes BRCA1 e BRCA2, se comparado as pacientes que teriam feito a SOOR bilateral profilática não teriam benefícios em relação a sobrevida global. Tendo o objetivo de prevenção ao desenvolvimento neoplásico a SOOR é beneficiada. Entretanto, deve avaliar a idade da paciente que será submetida a SOOR, e o risco e benéficos para esse aumento da taxa de sobrevida da paciente (ELEJE; EKE; EZEBIALU et al., 2018).

A adenomastectomia redutora de risco bilateral profilática é o método mais efetivo para reduzir o risco de câncer de mama entre portadoras da mutação nos genes BRCA1 e BRCA2 e o seu risco residual após a cirurgia é $<10 \%$. "Além da redução no risco de câncer de ovário em $90 \%$, demonstrou-se que a SOB reduz o risco de câncer de mama em, aproximadamente, $50 \%$ em portadoras prémenopáusicas de mutação que não tenham sido submetidas à cirurgia profilática" (COMANDER; CYGLER; RYAN, 2015, p. 749).

As pacientes que optarem por não realizar mastectomias profiláticas devem ser acompanhadas de perto com rastreamento para câncer de mama. De acordo com Comander, Cygler e Ryan (2015): 
As diretrizes para portadoras de mutação sugerem mamografias anuais, com início entre 25 e 30 anos de idade, exames clínicos da mama duas vezes ao ano e autoexame das mamas uma vez por mês. A ressonância magnética $(\mathrm{RM})$ da mama é mais sensível que a mamografia para detecção de câncer de mama em pacientes de alto risco, ao custo de maior taxa de resultados falso-positivos. As diretrizes da American Cancer Society para rastreamento de câncer de mama recomendam exame de RM anual associada à mamografia em pacientes portadoras de mutação em BRCA. As diretrizes clínicas sugerem iniciar com os exames de RM aos 25 anos de idade. Para as pacientes que optem por não realizar SOB profilática, recomenda-se rastreamento para câncer de ovário duas vezes ao ano com ultrassonografia transvaginal e dosagem de CA-125. Contudo, é necessário advertir que essas medidas não têm eficácia comprovada (COMANDER; CYGLER; RYAN, 2015)

A prevenção e o rastreamento de pacientes portadoras de mutações, nos referidos genes, devem ser realizados de maneira precoce. Após a confirmação diagnóstica, deve ser desenvolvido o manejo terapêutico mais eficiente para cada caso específico, abordando dentro desse panorama a importância da manutenção da qualidade de vida, bem estar biopsicossocial, retardando o aparecimento dos primeiros sinais e sintomas neoplásicos (PALUCH-SHIMON; CARDOSO; SESSA et al., 2016).

\section{Materiais e Métodos}

Trata-se de um recorte da pesquisa "Perfil epidemiológico e estimativa de sobrevida em pacientes portadores de câncer de mama e/ ou câncer de ovário com mutações gênicas BRCA1 e/ou BRCA2 submetidos a trata- mento oncológico", que é estudo de coorte retrospectiva. Os critérios de inclusão para a pesquisa foram o diagnóstico histopatológico de câncer de mama (CID C 50) e câncer de ovário (CID C 56) nos registros do Centro de Oncologia Clínica e Radioterapia Erechim. Os voluntários que participaram do estudo assinaram termo de consentimento livre e esclarecido, autorizando o uso dos dados constantes no instrumento de coleta de dados e a busca de informações em prontuários médicos. Os dados referentes às variáveis de pesquisa foram coletados com 32 pacientes através de entrevista, com aplicação de instrumento de coleta de dados desenvolvido, exclusivamente, para o projeto de pesquisa e revisão de prontuários clínicos no período de agosto/2019 a março/2020 no ambulatório do Centro de Oncologia Clínica e Radioterapia em Erechim, RS. Os resultados foram apresentados de forma descritiva e através de gráficos com as respectivas frequências das variáveis estudadas. O projeto atende as resoluções do CNS e foi aprovado pelo CEP URI Erechim.

\section{Resultados e Discussões}

Foram entrevistados 32 pacientes oncológicos portadores de câncer de mama e/ ou ovários que haviam sido testados para as mutações BRCA1 e BRCA2. Desses foram identificados 30 casos referentes ao diagnóstico de câncer de mama, um câncer de ovários e um caso de câncer de mama e ovários. Desta amostra, 31 são mulheres e um caso foi em homem, sendo este portador de câncer de mama. A média de idade entre eles foi de 60,70 anos, mediana 63 anos, desvio padrão 15,56 anos, idade mínima de 25 anos e idade máxima de 88 anos, demonstrando uma faixa etária relativamente jovem. Em coorte estudada nos EUA entre 2012 e 2019 e, publicação recente, observou-se que um quarto $(25,2 \%)$ de 187.535 
pacientes com câncer de mama e um terço (34,3\%) de 14.689 pacientes com câncer de ovário haviam sido testados, demonstrando a dificuldade de acesso aos testes genéticos, principalmente devido aos custos elevados. Sabemos que as mutações germinativas de BRCA1/2 são mais presentes nas jovens, sugerindo que, se possível, todas as pacientes com $<35$ anos sejam pesquisadas para a mutação (KURIAN; WARD; ABRAHAMSE et al., 2021).

Os resultados parciais referentes à variável de pesquisa, presença de histórico familiar de câncer, demonstraram a maior relação da ocorrência do câncer de mama nas pacientes com história prévia de neoplasia na família. Além disso, destaca-se a presença de outras neoplasias em alguns casos (Figura 3). Quanto à variável histórico de gestações, identificamos que $54,83 \%$ das pacientes portadoras das neoplasias relataram uma ou mais gestações. Apesar disso, a diferença do número de mulheres portadoras de câncer que não referem gestações é pequena (Figura 2). O principal tipo histológico detectado no grupo do câncer de mama foi o tipo ductal invasor representando $63,75 \%$ dos diagnósticos, seguido do lobular totalizando 9,3\%. Estes dados corroboram com dados da literatura mundial que estimam que os tipos histológicos ductais predominam entre as mulheres pré e pós menopausadas.

O estadiamento tumoral foi outra variável estudada e refere-se à presença de tumor $(\mathrm{T})$, acometimento de linfonodos $(\mathrm{N})$ e presença ou ausência de metástase (M) (TNM). A análise dos dados referente à variável estadiamento clínico tumoral demonstra a predominância do diagnóstico tardio do caso de câncer de mama e ovários, o que reduz significativamente a taxa de sobrevida em anos dos pacientes submetidos a tratamento (Figura 1). Além disso, as estratégias terapêuticas empregadas entre os participantes tiveram predominância da escolha de cirurgia para ressecção tumoral com uso de terapias adjuvantes, dentre elas, a quimioterapia, a radioterapia, e o uso de medicações hormonioterápicas e imunoterápicos. A modalidade de combinação de estratégias terapêuticas, utilizadas no grupo pesquisado das pacientes portadores de neoplasias de mama e/ou ovários, correspondem com a identificação de estágios mais avançados das neoplasias e a ocorrência de tumores em pacientes mais jovens. No grupo pesquisado, todos os pacientes $(100 \%)$ receberam mais de um tipo de modalidade de tratamento. A aplicação de terapias complementares à cirurgia, em caráter de adjuvância, em oncologia tem sido uma prática mundialmente recomendada a partir de critérios de estadiamento e perfil molecular e das neoplasias HENRY; SOMERFIELD; ABRANSON; et al., 2020).

A obesidade configura como fator de risco para o desenvolvimento do câncer de mama em mulheres pós menopáusicas, visto que o tecido adiposo passa a ser o principal tecido produtor de hormônios sexuais, principalmente estrogênio, levando também à resistência insulínica e sendo substrato para expressão de fatores de crescimento. Dessa forma as células estão mais suscetíveis a expressar os oncogenes. Além disso, outros fatores tem relação comprovada com o surgimento neoplásico, sendo eles a nuliparidade, menarca precoce e menopausa após os 50 anos (SILVA; PINHEIRO; RODRIGUES, et al., 2019). Neste estudo, a média de peso entre os participantes foi de $66 \mathrm{Kg}$, mediana de $65,5 \mathrm{Kg}$, desvio padrão de $11,5 \mathrm{Kg}$, peso mínimo no valor de $45,5 \mathrm{Kg}$ e máximo de $90 \mathrm{Kg}$. A média de estatura dos entrevistados resultou 1,62 metros, mediana 1,63 metros, desvio padrão de 8,27 , estatura mínima de 1,40 metros e máxima de 1,81 metros. Para o cálculo do índice de massa corporal foi utilizada a fórmula IMC: Peso/ altura ${ }^{2}$, para gerar a análise do gráfico (WHO, 2000). Os resultados demonstraram que $43 \%$ dos 
PERFIL EPIDEMIOLÓGICO DO CÂNCER DE MAMA E DE OVÁRIO: ANÁLISE DE DADOS PARCIAIS NUMA COORTE REGIONAL DE PACIENTES ONCOLÓGICOS

pacientes do grupo estudado apresentavam sobrepeso e/ou obesidade, no momento do diagnóstico, demonstrando relevância na análise desse fator de risco no desenvolvimento das neoplasias. (Figura 4).

Figura 1 - Distribuição dos pacientes da coorte estudada quanto ao Estadiamento (TNM)

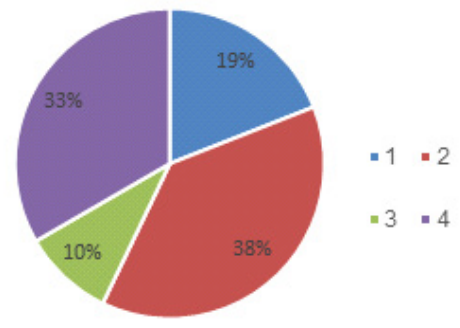

Figura 2 - Distribuição dos pacientes da coorte estudada quanto ao histórico familiar de gestação

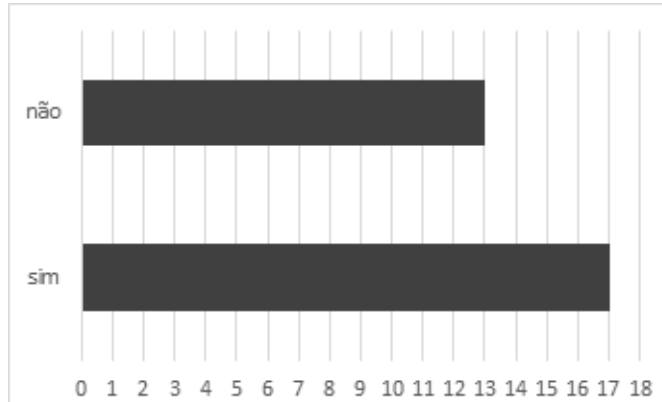

Figura 3 - Distribuição dos pacientes da coorte estudada quanto ao histórico familiar de câncer

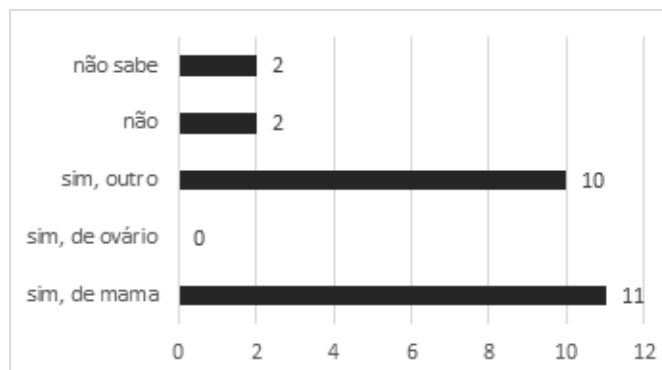

Figura 4 - Percentual de IMC dos pacientes da coorte estudada

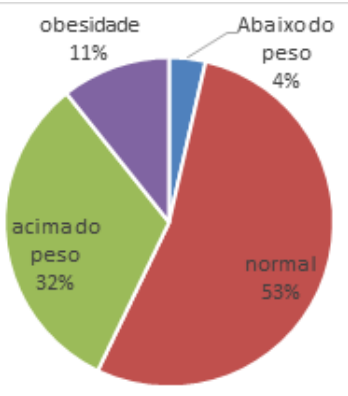

Figura 5 - Percentual de procedimentos realizados entre os pacientes da coorte estudada

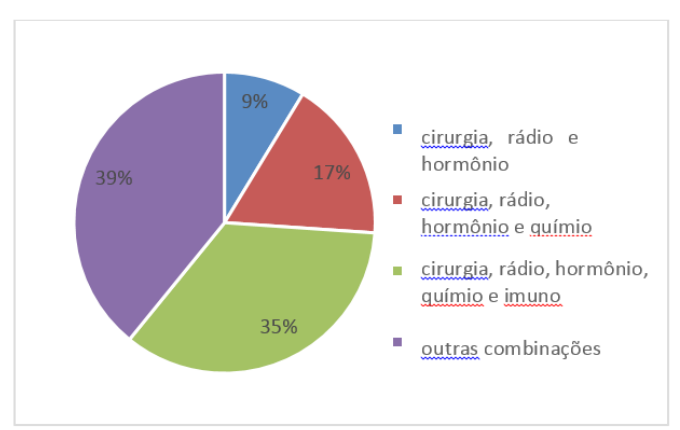

A prevenção do câncer de mama, por sua vez, pode ter diversas vertentes de atuação. Dessa forma, é possível pensar em intervenções que englobam toda a população e ações delimitadas para as mulheres que constituem grupo de risco para o desenvolvimento dessa neoplasia. A implementação de abordagens de prevenção que obtenham sucesso, requer, imprescindivelmente, que sejam realizadas a estratificação precisa do risco de câncer de mama, para que, dessa forma, as ações sejam precisamente direcionadas. (THORAT; BALASUBRAMANIAN, 2020). Recente metanálise demonstrou que o rastreamento mamográfico tem a capacidade de reduzir em $22 \%$ a mortalidade por câncer de mama. (DIBEN; OFFMAN; DUFFY et al., 2019). Dentre as estratégias de prevenção primária para o câncer de mama, destacam-se a manutenção de um peso corporal saudável, realização de 
atividade física regular, moderada ingestão de álcool e, em casos de mulheres que constituem grupo de alto risco, quimioprevenção utilizando medicamentos para redução de risco, como o anastrazol, tamoxifeno, raloxifeno e examestano. (COLDITZ; BOHLKE, 2014; VISVANATHAN; FABIAN; BANTUG et al., 2020)

Atualmente, o uso de biomarcadores tumorais séricos é a principal forma de rastreamento do câncer de ovário, sendo o CA 125 o mais utilizado, tanto para rastreamento quando para o follow-up da doença (LEDERMANN; RAJA; FOTOPOULOU et al., 2013). Apesar de ser uma glicoproteína expressa em diversos tecidos ovarianos, o CA125 ainda não faz parte de um consenso quanto ao rastreio das neoplasias ovariana, pois outras condições ginecológicas e reprodutivas podem aumentar os níveis séricos dessa proteína. Atualmente, o screening de câncer de ovário é multimodal, utilizando de ultrassonografia transvaginal ou pélvica, além de outros métodos de imagem para corroborar com o diagnóstico, como tomografia, ressonância magnética e tomografia com emissão de pósitrons (PET-CT) (CONITEC, 2019).

\section{Conclusões}

O teste genético é cada vez mais valioso para a avaliação do risco de câncer, avaliação de triagem, estratégias de redução de risco e seleção de tratamento. A inovação em tecnologia de sequenciamento e declínio dos custos para realização de exames poderão facilitar a utilização dos testes genéticos na abordagem do tratamento do câncer. Este artigo de revisão, que incluiu uma breve descrição epidemiológica das características dos pacientes portadores de neoplasia de mama e de ovário, apesar das limitações de ser uma análise parcial e de ser composta por um número pequeno de pacientes, ratificou que o câncer de mama continua sendo o mais prevalente na população feminina, em relação aos casos de câncer de ovários. $\mathrm{O}$ tipo histológico carcinoma ductal invasivo da mama foi o mais frequente na amostra estudada e os diagnósticos foram realizados em estágios mais avançados, sendo que tais características dificultam o manejo adequado e precoce da doença e, consequentemente, impacta na redução da taxa de sobrevida. Além disso, o presente estudo reforça a importância do conhecimento das mutações nas linhagens genéticas dos genes $B R C A 1$ e $B R C A 2$, a fim de aprimorar a promoção, a prevenção e a seleção de tratamentos dos pacientes portadores de neoplasias de mama e ovários e de seus familiares. 
PERFIL EPIDEMIOLÓGICO DO CÂNCER DE MAMA E DE OVÁRIO: ANÁLISE DE DADOS PARCIAIS NUMA COORTE REGIONAL DE PACIENTES ONCOLÓGICOS

\section{REFERÊNCIAS}

BRAY, F.; FERLAY, J.; SOERJOMATAR, A.M.; SIEGEL, R.L.; TORRE, L.A.; JEMAL, A. Global Cancer Statistics 2018: GLOBOCAN. Estimates of Incidence and Mortality Worldwide for 36 Cancers in 185 Countries. CA: a Cancer Journal for Clinicians, v. 68, n. 6, p. 394-424, 2018.

COMANDER. A; CYGLER. T; RYAN. D.P; Oncologia da mama: apresentação clínica e genética. In: CHABNER, Bruce A.; LONGO, Dan L. Manual de Oncologia de Harrison. 2. ed. Cap. 57. Porto Alegre: Artmed, 2015

COELHO, A. S.; SANTOS, M.A.S.; CAETANO, R. In. PIOVESAN, C.F.; FIUZA, L.A.; MACHADO, R. L. D.; FURINI, A.A. D.C. Predisposição hereditária ao câncer de mama e sua relação com os genes BRCA1 e BRCA2: revisão da literatura. Revista Brasileira de Análises Clínicas. 2018. Disponível em:http://www.rbac.org.br/wp-content/uploads/2018/06/RBAC-vol-501-2018-ref-615.pdf

COLDITZ, G. A.; BOHLKE, B. Priorities for the Primary Prevention of Breast Cancer. CA: A Cancer Journal for Clinicians. United States, v. 64, p. 186-194, 2014.

CONITEC. Diretrizes Diagnósticas e Terapêuticas de Neoplasia maligna epitelial de ovário. Ministério da saúde, Brasília-DF. Janeiro, 2019. Disponível em: http:/conitec.gov.br/images/ Relatorios/2019/Relatorio_DDT_NeoplasiaMalignaEpitelialdeOvario_2019.pdf

DIBEN,A.; OFFMAN, J.; DUFFY,S. W.; GABE, R. Worldwide review and meta-analysis of cohort studies measuring the effect of mammography screening programmes on incidence-based breast cancer mortality. Cancers, v.12, Issue 4, 2020.

DOWNS, BRADLEY; WANG, SAN MING. Epigenetic changes in BRCA1-mutated familial breast cancer. Cancer Genetics. Elsevier, v. 208, Issue 5, p.237-240, 2015.

DUFLOTH, R.M; CARVALHO, S.; HEINRICH, J. K.; SHINZATO, J. Y.; SANTOS. C.C.D.; ZEFERINO, L.C.; SCHIMITT, F. Analysis of BRCA1 and BRCA2 mutations in Brazilian breast cancer patients with positive family history. São Paulo Medical Journal. 2005.

ELEJE, G.; EKE, A.; EZEBIALU, I.; IKECHEBELU, J.; UGWU, E.O.; OKONKWO, O.O. Riskreducing bilateral salpingo-oophorectomy in women with BRCA1 or BRCA2 mutations. The Cochrane database of systematic reviews, v. 8, Issue 8, 2018.

HENRY, N.; SOMERFIELD, M.; ABRANSON, V. et al. Role of patient and disease factors in adjuvant systemic therapy decision making for early-stage, operable breast cancer: Update of the ASCO endorsement of the cancer care Ontario guideline. Journal of clinical oncology, v. 37, Issue 22, p. 1965-1977.

HONRADO, EMILIANO; BENÍTEZ, JAVIER; PALACIOS, JOSÉ. Histopathology of BRCA1- and BRCA2-associated breast cancer. Critical Reviews in Oncology/Hematology. Elsevier, v. 59, Issue 1, p. 27-39, 2006.

INSTITUTO NACIONAL DO CÂNCER JOSÉ DE ALENCAR GOMES DA SILVA. Estimativa 2020, câncer no Brasil. 2019. Disponível em: https:/www.inca.gov.br/sites/ufu.sti.inca.local/files/ media/document/estimativa-2020-incidencia-de-cancer-no-brasil.pdf. Acesso em: 31 maio 2020.

KUCHENBAECKER, K.; HOPPER. J.; BARNES. D.; PHILLIPS, A.K.; MOOJ, T.M.; ROSS-BLOOM, M.; JERVIS, S.; VAN LEEUWEN, F. E.; MILNE, R.L.; ANDRIEU, N.; GOLDGAR, D.E.; TERRY, M.B.; ROOKUS, M.A.; EASTON, D.F. ANTONIOU, A.C.; Risk of breast, ovarian and contralateral breast cancer for BRCA1 and BRCA2 mutation carrier. Jama, v. 317, issue 23, 2017. 
Emilli Cristina Dos Santos - Juliano Sartori - Tayná Andressa Wencelewski - Nicole De Albuquerque - Franciele Dartora - Gabriela Bassani Fahl Rafael Rossa Marsarotto - Paola Wozniak Ceni - Bruna Albiero Decesaro -Milena Bitarello - Luma Girardi Kautz - Amanda Peracchi Schneider

KUMAR, V.; ABBAS, A. K.; FAUSTO, N. Robbins e Cotran Patologia: bases patológicas das doenças. 8. ed. Rio de Janeiro: Elsevier, 2010.

KURIAN, W.A.; WARD, K.C.; ABRAHAMSE, P.; BONDAREK, I.; HAMILTON, A.S.; DEAPEN, D.; MORROW,M.; BERK, J.S, HAFER, T.P.; KATZ, S. Time Trends in Receipt of Germline Genetic Testing and Results for Women Diagnosed With Breast Cancer or Ovarian Cancer, 2012-2019. Journal of Clinical Oncology., p. 1-10, February, 2021.

LANCASTER, M.J.; POWELL, C.B.; KAUFF, N.D.; CASS, I.; CHEN, L.; LU, H.K.; MUTCH, G.D.; BERCHUCK, A.; KARIAN, B.Y.; HERZOG, T.J. Society of Gynecologic Oncologists Education Committee statement on risk assessment for inherited gynecologic câncer predispositions. Gynecol Oncol, v. 107, Issue 2, p. 159-162, 2007.

LEDERMANN, J.A.; RAJA, F.A; FOTOPOULOU, C.; GONZALES-MARTINS, A.; COLOMBO, N.; SESSA, C. Newly diagnosed and relapsed epithelial ovarian carcinoma: ESMO Clinical Practice Guidelines for diagnosis, treatment and follow-up. Annals of Oncology, v. 24, Supplement 6, p. vi24-vi32, 2013.

MORI, H.; KUBO, M.; KAI ,M.; VELASQUEZ, V.V.; KURATA, K.; YAMADA, M.; OKIDO, M .I.; KUROKI, S.; ODA,Y. I.; NAKAMURA, M. BRCAness Combined With a Family History of Cancer Is Associated With a Poor Prognosis for Breast Cancer Patients With a High Risk of BRCA Mutations. Clinical Breast Cancer. Elsevier, v.18, Issue 5, p. e1217-e1227, 2018.

MOSCHETTA, M.; GEORGE, A.; KAYE, S. B.; BANERJEE, S. BRCA somatic mutations and epigenetic BRCA modifications in serous ovarian cancer. Ann Oncol., v. 27, n. 8, p.1449-1455, 2016.

NATIONAL INSTITUTE FOR HEALTH AND CARE EXCELLENCE. Familial breast cancer: Classification and care of people at risk of familial breast cancer and management of breast cancer and related risks in people with a family history of breast cancer. 2013. Disponível em: https://www. nice.org.uk/guidance/cg164/resources/familial-breast-cancer-classification-care-and-managingbreast-cancer-and-related-risks-in-people-with-a-family-history-of-breast-cancer-pdf-35109691767493 . Acesso em: 31 maio 2020.

PALUCH - SHIMON S.; CARDOSO, F.; SESSA, C.; BALMANA, J.; CARDOSO, J. M.; GILBERT, F.; SENKUS, E. Prevention and screening in BRCA mutation carriers and other breast/ ovarian hereditary cancer syndromes: ESMO Clinical Practice Guidelines for cancer prevention and screening. Annals of Oncology, v.27, Supplement 5, p. v103-v110, 2016.

REBBECK, T.; MITRA, N.; WAN, F.; SINILNIKOV. O.M.; HEOLEY, S.; MC GUFFOLF, L.; CHENEVIX - TRENCH, G.; EASTON, D.F.; ANTOIOU, A.C.; NATHANSON, K.L.. Association os type and location of BRCA1 and BRCA2 mutations with risk of breast and ovarian câncer. Jama, v. 313, 2015.

SILVA, A; PINHEIRO, G; RODRIGUES, E.; SANTOS, W.D.S.; ARAUJO, C..M.D.S.; ARAUJO, R.D.S; SILVA, C.A.D.; LEITE, F.D.M. C.; CUNHA, K .J .B. FATORES PREDISPONENTES PARA O CÂNCER DE MAMA E QUALIDADE DE VIDA: REVISÃO INTEGRATIVA. Brazilian Journal Of Surgery and Clinical Research, v.27, p. 148- 153. 2019.

SHIOVITZ, S.; KORDE, L. A. Genetics of Breast Cancer: a topic in evolution. Annals of Oncology, v. 26, p.1291-1299, 2015

SØNDERSTRUP, I.; JENSEN, M.R.; EJLERTSEN, B.; ERIKSEN, J.D.; GERDES, A.; KRUSE, T.A; LARSEN, M.J.; THOMASSEN, M.I.; LÆNKHOLM, A. Subtypes in BRCA-mutated breast cancer. Human Pathology. Elsevier, v. 84, p. 192-201, 2019

SØRENSEN, CLAUS STORGAARD. Hereditary Risk of Breast and Ovarian Cancer: BRCA1 and BRCA2. Encyclopedia of Cancer. Elsevier, p. 214-217, 2019 
THORAT, M. A.; BALASUBRAMANIAN, R. Breast cancer prevention in high-risk women. Best Practice \& Research Clinical Obstetrics and Gynaecology, v. 65, p. 18-31, 2020.

VIEIRA, R. A. C.; BILLER, G.; UEMURA, G.; RUIZ, C. A.; CURADO, M. R.. Breast cancer screening in developing countries. Clinics, v. 72, n. 4, p. 244-253, 2017.

VISVANATHAN, K.; FABIAN, C.; BANTUG, E.; BREWSTER, A.M.; DAVIDSON, N.E.; DE CENSI, A.; FLOYD, J.D.; GARBER, J.E.; HOFSTATTER, E.W.; KHAM, S.; KATAPODI, M.C.; PRUTHI, S.; RAAB, R.; RUNOWICZ, C.D.; SOMERFIELS, M.R. Use of Endocrine Therapy for Breast Cancer Risk Reduction: ASCO Clinical Practice Guideline Update. Journal of clinical oncology, v. 37, Issue 33, p. 3152-3165, 2019.

WORLD HEALTH ORGANIZATION. Obesity: preventing and managing the global epidemic. Report of a World Health Organization Consultation. Geneva: World Health Organization, 2000. 253 p. (WHO Obesity Technical Report Series, n. 894). Disponível em: http://www.who.int/ nutrition/publications/obesity/WHO_TRS_894/en/ Acesso em: 09 ago. 2020. 
